\title{
Development of a Mid-Infrared Laser for Study of Infrared Countermeasures Techniques
}

\author{
H.H.P.Th. Bekman ${ }^{1}$, J.C. van den Heuvel, F.J.M van Putten, H.M.A. Schleijpen \\ TNO- Physics and Electronics Laboratory, \\ P.O. Box 96864, 2509 JG The Hague, The Netherlands
}

\begin{abstract}
Countermeasures against heat seeking missiles require access to efficient laser sources, which should emit wavelengths at band I, II and IV. Efficient diode pumped solid-state lasers, combined with efficient non-linear wavelength shifters, allow the development of practical tuneable mid-IR countermeasure sources. The paper describes the requirements and the development of a tabletop laser source for study of DIRCM techniques. Jamming laser systems must be able of creating pulse sequences in the frequency range between $100 \mathrm{~Hz}$ and $10,000 \mathrm{~Hz}$, including the capability to mix and sweep the jam frequency. A Nd: $\mathrm{YVO}_{4}$ pump laser with maximum pump power of 3 Watt and pulse length of $10 \mathrm{~ns}$, and a maximum modulation frequency of $100 \mathrm{kHz}$ was selected. A linear single resonant OPO cavity with $30 \mathrm{~mm}$ long, $1 \mathrm{~mm}$ thick PPLN crystals was build. With the tabletop laser system we were able to generate wavelengths from 1.5 to 4 micron. In band I, at 2 micron we can generate between 400-550 mW, and in band II, from 3-4 micron we can generate 130-160 mW laser jam power. The beam quality $\left(M^{2}\right)$ is approximately 2.5 . The power efficiency for the idler was $8.8 \%$, while the slope power efficiency was $15 \%$. Jam patterns are generated by use of an acousto-optic modulator.
\end{abstract}

Keywords: OPO, Nd:YVO4, PPLN, Laser, MID-IR, Countermeasures

\section{INTRODUCTION}

The first project to study laser-jamming technology started at TNO-FEL in 1995, after initial assessment of the vulnerability of passive sensors. A solid-state laser source radiating at $4 \mu \mathrm{m}$ has been developed in that project. A follow-up project was defined in which the laser source was used to study damage of IR sensors at close range using strongly focused laser beams. The confidence gained from these laboratory experiments has initiated the current project. In this project a new laser source for jamming experiments has been developed in a laboratory set-up. Under certain provisions, this set-up can be used in field experiments. With the availability of a laser jamming source there is the capability to generate jamming patterns to study and test the jamming of infrared guided missiles. For dazzling and damaging more powerful lasers have to be developed.

In Section 2 of this paper the laser requirements are derived from available DIRCM system requirements. Based on the requirements, the components for the laser are selected. Section 3 describes the construction and characterisation of the assembled DIRCM laser. The laser is built using solid state laser technology using non-linear frequency conversion in an optical parametric oscillator. With the development of a laser jamming system the capability to generate and investigate jam patterns against various seekers will become available for the Royal Netherlands Air Force.

Heat-seeking missiles like the Stinger, Sidewinder, and others are an ever-increasing threat for fixed-wing and rotarywing aircraft. The traditional countermeasures like maneuvering and flares have a reduced effectiveness against modern missiles. The experience of Desert Storm has shown that from all US aircraft most losses fell to IR SAM missiles.

In the Kosovo crises air campaigns were conducted at high altitude to reduce this risk, but this has also reduced the effectiveness of air strikes. In addition to the development of advanced decoy flares and infrared jammers also a new method is being developed to counter heat-seeking missiles. It is based on active sources that deceive, dazzle or (sensor) damage these missiles.

\footnotetext{
${ }^{1}$ bekman@fel.tno.nl
} 
Practical use of such countermeasures, therefore, requires access to efficient laser sources, which should preferably be tuneable to any wavelength within the 3-5 $\mu \mathrm{m}$ spectral band. In the past there has been a lack of such sources. However, the advent of efficient diode pumped solid-state lasers, combined with efficient non-linear wavelength shifters, has recently paved the way for development of practical tuneable mid-IR countermeasure sources, and the first of such systems are about to be installed on airborne platforms for protection against IR homing missiles.

\section{LASER SPECIFICATION DERIVED FROM DIRCM REQUIREMENTS}

Optical parametric oscillators (OPOs) are the laser sources of choice when needing broad continuous tunablility, high peak power $(>1 \mathrm{~kW})$, and high $(>30 \%)$ conversion efficiency. It is generally adopted that a jammer to signal $(\mathrm{J} / \mathrm{S}) \mathrm{ratio}$ of $>30 \mathrm{~dB}$ is required. For the $3-5 \mu \mathrm{m}$ band reference values for the aircraft signatures are in the range $10-1000 \mathrm{~W} / \mathrm{sr}^{1,2}$ depending on aspect angle.

The directed energy of the laser reduces the required jammer energy in comparison to a lamp based jammer system. The required laser power is therefore a function of the laser divergence. In most Directed Infrared Countermeasure (DIRCM) system designs, an infrared Focal Plane Array (FPA) camera is used to passively track the threat. These cameras typically have a field of view (FOV) of $4 \times 4^{\circ}$ and $256 \times 256$ pixels, which yields an instantaneous field of view (IFOV) resolution of $0.3 \mathrm{mrad}$. The divergence of the laser beam must be in balance with this spatial resolution of the tracking camera. The laser beam divergence also needs to be compatible with the update rate of the camera (typically $400 \mathrm{~Hz}$ ) to reach the required time on target even when the missile or the platform is manoeuvring.

Furthermore, we must keep in mind the fact that the camera will track the plume of the threat missile, whereas the jamming beam must be pointed at the missile seeker head. Given a typical missile length of $2 \mathrm{~m}$, seen at an aspect angle of $30^{\circ}$, the separation of aim point and tracking point, projected perpendicular to the line of sight, can be as large as $1 \mathrm{~m}$. At $1000 \mathrm{~m}$ this is equivalent to $1 \mathrm{mrad}$. Based on the above a laser beam divergence between 1 and $2 \mathrm{mrad}$ seems adequate.

For a $1 \mathrm{mrad}$ laser beam divergence, the required laser power is $1 \mathrm{~W}$. This is based on a solid angle of $10^{-6} \mathrm{sr}(1 \mathrm{mrad}$ beam divergence) an aircraft signature of $1000 \mathrm{~W} / \mathrm{sr}$ and a $J / S$ ratio of 1000 .

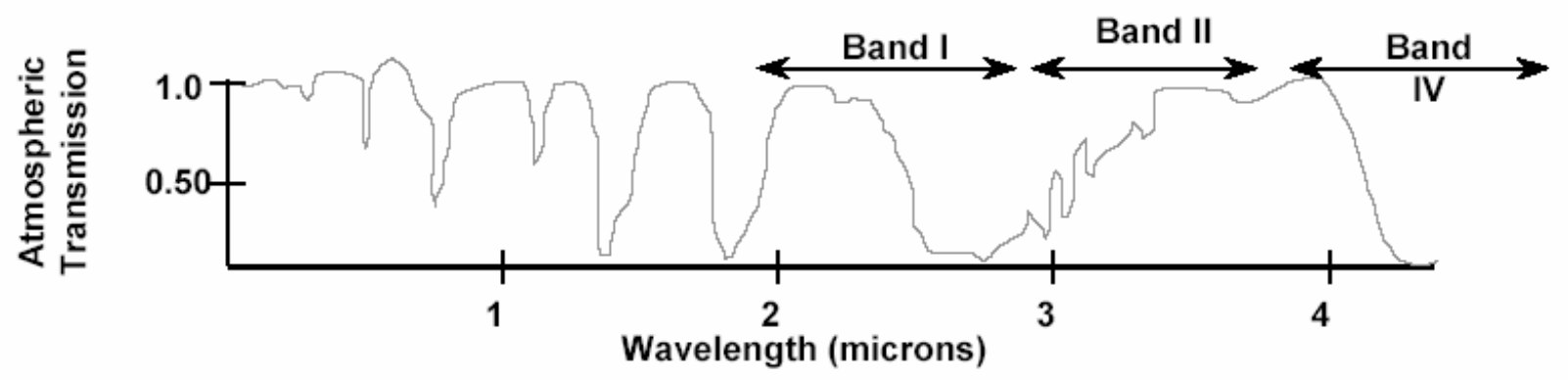

Figure 1 Wavelengths bands commonly used by seekers

The laser has to operate in three wavelength bands, which are depicted in Fig. 1. These bands are selected since most of the IR guide missiles make use of one of these bands. The missiles use these bands since air targets are very hot and emit a lot of thermal radiation in these bands. Furthermore, the atmospheric transmission is relatively good.

In addition, detectors in these wavelength bands are available. For the 1-3 $\mu \mathrm{m}$ band uncooled PbS detector cells are used, whereas for the 3-5 $\mu \mathrm{m}$ band cooled PbSe or InSb detectors are used. The more advanced seekers use the 3-5 $\mu \mathrm{m}$ band. 
In adverse weather the jamming laser will experience stronger attenuation in the atmosphere. This affects the maximum range at which it can deceive an incoming IR guided missile. At the same time, the missiles also have more difficulty in acquiring their targets, since they experience a stronger atmospheric extinction of their target signal as well. Thus in effect, the laser jamming system is not sensitive to weather/environmental conditions as far as attenuation is concerned, since the jamming laser and the IR guided missile experience the same attenuation losses.

Wavelengths above $4 \mu \mathrm{m}$ are more difficult to generate since single, PPLN based, OPO systems have severe absorption losses above $4 \mu \mathrm{m}$. Therefore, a more complex tandem OPO system has to be used or a single OPO system with a less powerful laser pump source.

It can be shown that an effective jamming source needs to generate modulated infrared light. The jamming modulation frequency/pattern has to be similar to the modulation patterns that the reticle/conscan/rosette-scan seeker generates out of the target radiation.

We know from various sources that jamming laser systems must be able to create pulse sequences in the frequency range typically between $100 \mathrm{~Hz}$ and $10,000 \mathrm{~Hz}$, including the capability to mix and sweep the jam frequency. These high frequencies dictate that diode pumped laser pump sources have to be used, since flash lamp pumped laser pumps do not operate well above approx. a few hundred Hertz.

As OPO lasers make use of a non-linear optical crystal, high peak powers are required. Therefore, pulsed pump lasers are used. The laser light is stored in an optical cavity and released in a very short intense burst of light. Also, the midinfrared light generated in the OPO light is therefore pulsed. The laser pulse length can vary, but is typical in the 1$100 \mathrm{~ns}$ regime. The pulse length needed for the jam patterns is normally much longer than these laser pulse lengths. Therefore, a jamming pulse has to be created by filling a jam pulse with a number of laser pulses as shown in Fig. 2. The time delay introduced in the seeker electronics smears out the individual laser pulses to the required jam pulse.

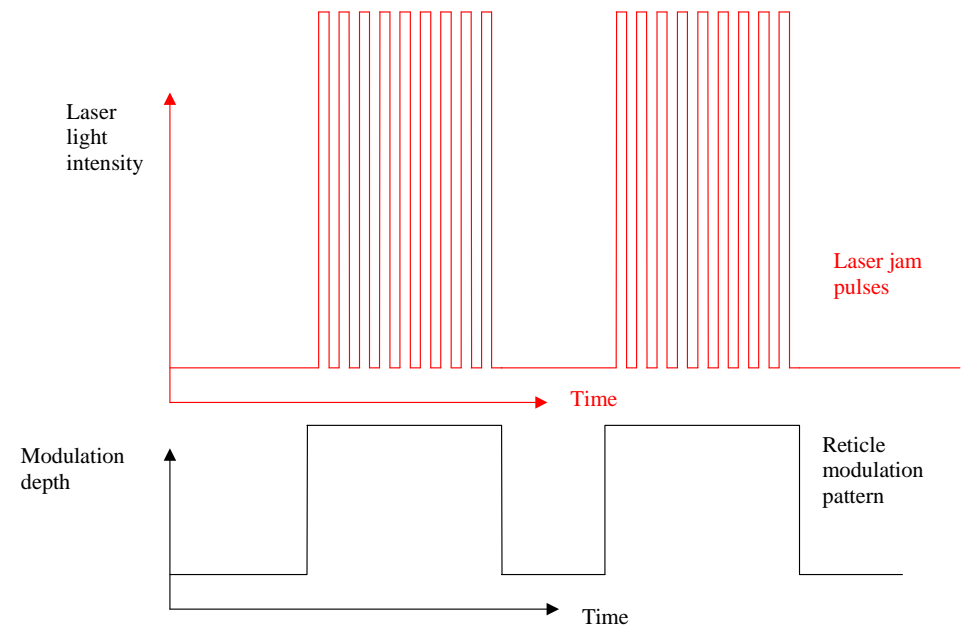

Figure 2 Laser jam source intensity versus time and reticle modulation pattern

In summary the operational laser requirements are provided in the following table:

Table 1: General operational DIRCM laser requirements.

\begin{tabular}{ll}
\hline Wavelength & $2-5 \mu \mathrm{m}$ \\
Modulation frequency & $20-50 \mathrm{kHz}$ \\
\hline
\end{tabular}

In case of passive tracking a laser beam divergence between 1 and 2 mrad is most realistic ${ }^{2}$. The required laser power in this case is summarised in the Table 2. 
Table 2: Required laser power for an aircraft signature of 10 or $1000 \mathrm{~W} / \mathrm{sr}$ a J/S ratio of $30 \mathrm{~dB}$ and a beam divergence of 1 and 2 mrad.

\begin{tabular}{lll}
\hline & $10 \mathrm{~W} / \mathrm{sr}$ & $1000 \mathrm{~W} / \mathrm{sr}$ \\
\hline $1 \mathrm{mrad}$ & $10 \mathrm{~mW}$ & $1000 \mathrm{~mW}$ \\
$2 \mathrm{mrad}$ & $40 \mathrm{~mW}$ & $4000 \mathrm{~mW}$ \\
\hline
\end{tabular}

The required beam quality depends on the optical aperture that is used. If we assume that we can have an aperture that allows a beam diameter of 15 or $25 \mathrm{~mm}$, we can calculate the minimum required beam quality to arrive at a beam divergence of 1 or $2 \mathrm{mrad}$, see Table 3.

Table 3: Maximum allowed beam quality $\left(M^{2}\right)$ for a laser beam at $\lambda=5 \mu \mathrm{m}$, and for a required laser beam divergence of 1 or $2 \mathrm{mrad}$ with an initial optical beam width of 15 or $25 \mathrm{~mm}$.

\begin{tabular}{lll}
\hline $\mathrm{M}^{2}$ & $1 \mathrm{mrad}$ & $2 \mathrm{mrad}$ \\
\hline $15 \mathrm{~mm}$ & $<2.3$ & $<4.7$ \\
$25 \mathrm{~mm}$ & $<3.9$ & $<7.9$ \\
\hline
\end{tabular}

It is not our objective to build a jam laser that will be incorporated in a flyable operational DIRCM system. The laser will be used to study infrared countermeasure techniques in laboratory experiments and during field trials. Therefore it does not have to be as powerful as indicated in the above tables, which were derived for an operational system.

\section{CONSTRUCTION AND CHARACTERISATION OF LASER SOURCE}

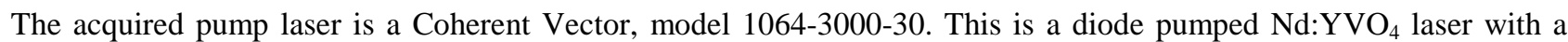
specified maximum average output of $3 \mathrm{Watt}$. At $30 \mathrm{kHz}$ the pulse energy is approx. $100 \mu \mathrm{J}$. At higher pulse rates the pulse energy decreases, whereas at lower pulse rates the average power decreases but pulse energy increases (see Table 4).

Table 4: Measured characteristics of Coherent Vector pump laser.

\begin{tabular}{|c|c|c|c|c|c|}
\hline $\begin{array}{l}\text { Rep } \\
(\mathrm{kHz})\end{array}$ & rate & $\begin{array}{l}\text { Pulse energy } \\
(\mu J)\end{array}$ & $\begin{array}{l}\text { Average power } \\
\text { (W) }\end{array}$ & Peak power $(\mathrm{kW})$ & $\begin{array}{l}\text { Pulse length } \\
\text { (ns) }\end{array}$ \\
\hline 5 & & 288 & 1.44 & 57.6 & 5.0 \\
\hline 10 & & 160 & 1.60 & 30.2 & 5.3 \\
\hline 15 & & 132 & 1.98 & 17.6 & 7.5 \\
\hline 20 & & 118 & 2.35 & 14.4 & 8.2 \\
\hline 30 & & 103 & 3.1 & 11.2 & 9.2 \\
\hline 40 & & 78 & 3.1 & 6.78 & 11.5 \\
\hline 50 & & 62 & 3.1 & 4.43 & 14 \\
\hline 60 & & 52 & 3.1 & 3.25 & 16 \\
\hline 70 & & 44 & 3.1 & 2.59 & 17 \\
\hline 80 & & 39 & 3.1 & 2.05 & 19 \\
\hline 90 & & 34 & 3.1 & 1.70 & 20 \\
\hline 100 & & 31 & 3.1 & 1.35 & 23 \\
\hline
\end{tabular}

A multi-grating PPLN crystal manufactured by Deltronic is used. The crystals have a thickness of $1 \mathrm{~mm}$ and a length up to $50 \mathrm{~mm}$. For the laser 2 crystals with identical grating periods were available, with a length of $20 \mathrm{~mm}$ and $30 \mathrm{~mm}$. These crystals are divided into 8 separate sections, each with a different grating period.

The PPLN crystal is placed in an oven (Figure 3), which has two reasons. PPLN crystals can be damaged by high power green radiation. Due to the high gain in the crystal and the high laser power, frequency doubling of the pump to $532 \mathrm{~nm}$ (green light) takes place as well, in addition to OPO conversion. With the crystal at an elevated temperature, photorefractive damage can be suppressed. Secondly, a change in crystal temperature changes the wavelength of the OPO output. 


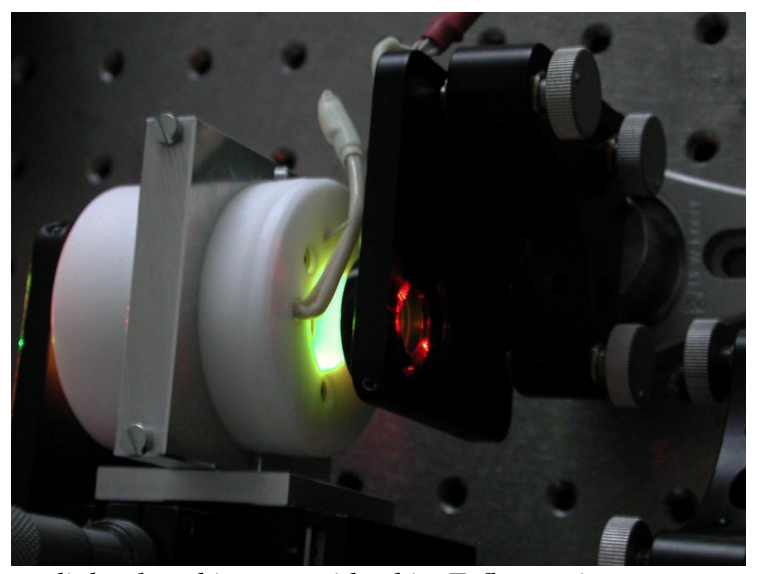

Figure 3 PPLN crystal emitting green light placed in oven with white Teflon casing.

Based on the results of Arisholm ${ }^{3}$ we decided to use a crystal temperature of $190^{\circ} \mathrm{C}$ in our experiments as previously described. The tuning curve of the PPLN OPO, when kept at a temperature of $190^{\circ} \mathrm{C}$ and pumped with $1.064 \mu \mathrm{m}$, is drawn in Figure 4. The vertical lines show the 8 crystal periods of the two crystals we have acquired.

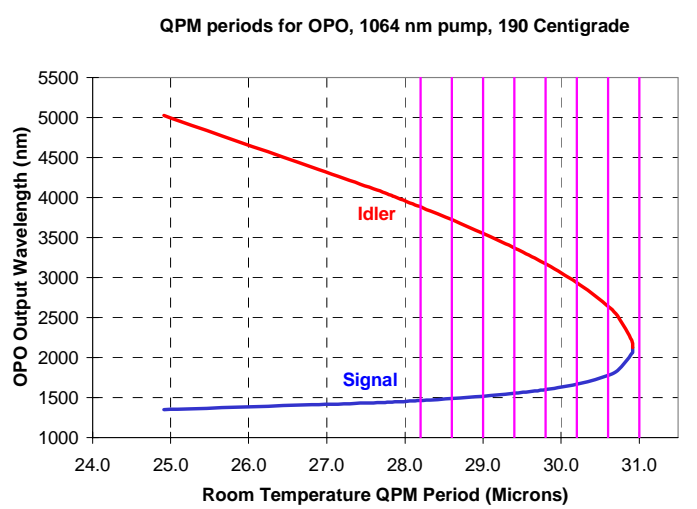

Figure 4 Required PPLN period for different wavelengths

In Table 5 the wavelength range for the different grating periods (with grating period specified at room temperature RT) of the PPLN crystal is given at $100^{\circ} \mathrm{C}$ (about the lowest crystal temperature found in our literature survey), $190^{\circ} \mathrm{C}$ and $220^{\circ} \mathrm{C}$ (maximum oven temperature).

Table 5 Wavelength range of PPLN crystal.

\begin{tabular}{llllllll}
\hline & RT period & \multicolumn{3}{l}{ Signal $(\mathrm{nm})$} & \multicolumn{3}{l}{ Idler $(\mathrm{nm})$} \\
pump $(\mathrm{nm})$ & $(\mu \mathrm{m})$ & $100^{\circ} \mathrm{C}$ & $190^{\circ} \mathrm{C}$ & $220^{\circ} \mathrm{C}$ & $100^{\circ} \mathrm{C}$ & $190^{\circ} \mathrm{C}$ & $220^{\circ} \mathrm{C}$ \\
\hline 1064 & 28.20 & 1447 & 1466 & 1474 & 4020 & 3880 & 3825 \\
1064 & 28.60 & 1468 & 1490 & 1500 & 3870 & 3722 & 3661 \\
1064 & 29.00 & 1491 & 1519 & 1531 & 3715 & 3552 & 3488 \\
1064 & 29.40 & 1519 & 1555 & 1571 & 3552 & 3370 & 3297 \\
1064 & 29.80 & 1553 & 1601 & 1625 & 3379 & 3172 & 3082 \\
1064 & 30.20 & 1597 & 1669 & 1707 & 3187 & 2935 & 2825 \\
1064 & 30.60 & 1658 & 1780 & 1880 & 2969 & 2645 & 2451 \\
1064 & 31.00 & 1756 & $*$ & $*$ & 2700 & $*$ & $*$ \\
\hline
\end{tabular}

*: no phase matching possible with this period above $\sim 175^{\circ} \mathrm{C}$ 
With the pump laser and the PPLN crystal a laser was built (see Figure 5). A linear cavity design was used.

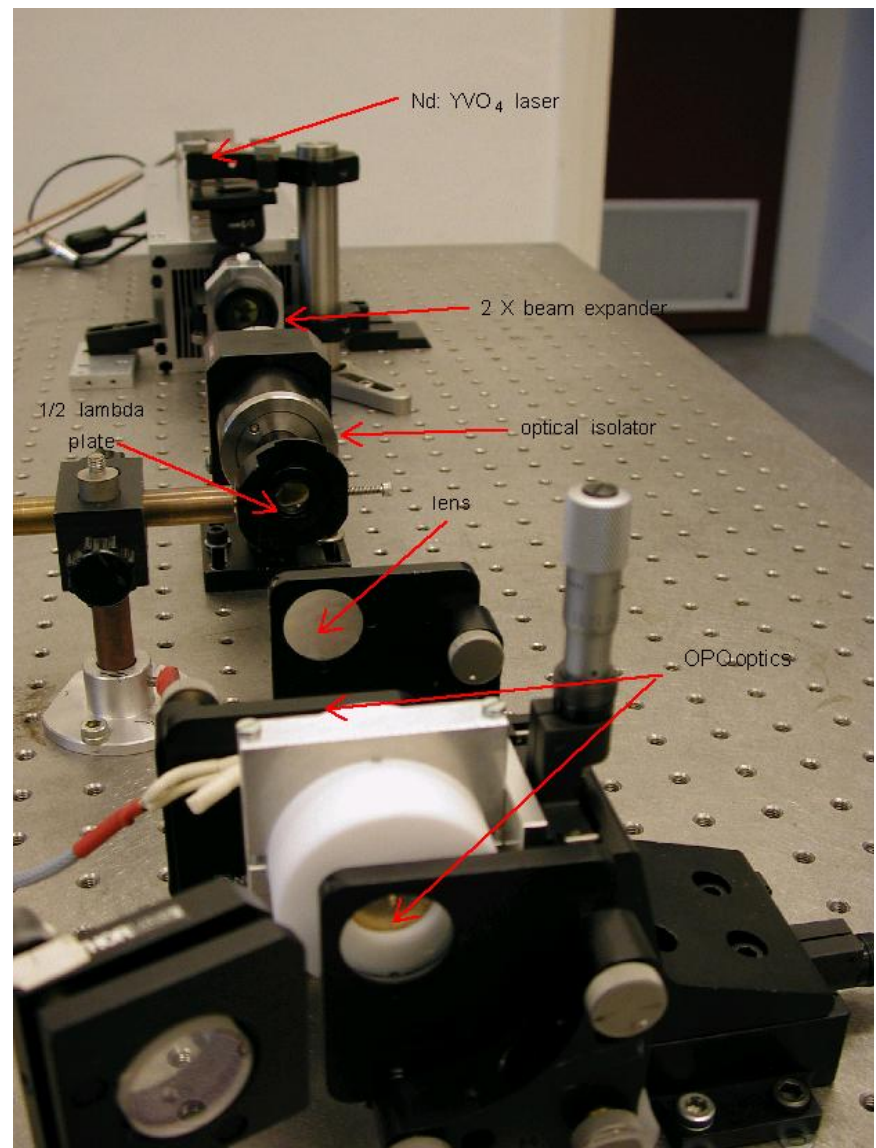

Figure 5 Overview laser on bread board

The pump laser beam diameter is increased to approx. $2 \mathrm{~mm}$ with a beam expander. An acousto-optic modulator is then used for modulation of the pump pulse train (not shown in Figure 5). Next, the pump laser beam goes through an optical isolator, so that reflections from optical components further on cannot damage the pump laser or the beam expander. A $1 / 2 \lambda$ wave plate is used to adjust the polarisation of the pump laser beam. Non-linear generation in the PPLN crystal only occurs for a single linear polarisation. A lens with a focal length of $100 \mathrm{~mm}$ or $150 \mathrm{~mm}$ focuses the pump laser beam in the centre of the PPLN crystal. The crystal is placed in a linear cavity. The input coupler (first mirror of cavity) has a high transmission for the pump $(1064 \mathrm{~nm})$ and a high reflection for the generated signal. The output coupler (second mirror of the cavity) has a reflection of about $15 \%$ to $20 \%$ for signal and idler and approx. $10 \%$ for the pump. Appropriate band pass filters after the cavity can be used to separate the pump, signal and idler wavelengths.

According to the manufacturer (Deltronic), the maximum allowed power density on the PPLN crystal surface is $300 \mathrm{MW} / \mathrm{cm}^{2}$. With the $F=100 \mathrm{~mm}$ lens the power density in the focus is (with a $20 \mathrm{kHz}$ rep-rate, $100 \mu \mathrm{J}$ energy and $8.2 \mathrm{~ns}$ pulse length): $275 \mathrm{MW} / \mathrm{cm}^{2}$, close to the maximum power density. However, this is not on the surface, but inside the PPLN crystal, in which damage thresholds are usually much higher. Even so, care must be taken that the laser is not focused on crystal surfaces, especially with the $F=100 \mathrm{~mm}$ lens.

The $30 \mathrm{~mm}$ PPLN OPO has been tested with two different lenses, with a focal length of $100 \mathrm{~mm}$ and $150 \mathrm{~mm}$. The temperature of the $30 \mathrm{~mm}$ long crystal was kept at $190^{\circ} \mathrm{C}$ for all experiments unless mentioned otherwise. In Figure 6 the generated idler output for these two situations are shown at $20 \mathrm{kHz}$ laser pump rep-rate as function of pump power. 


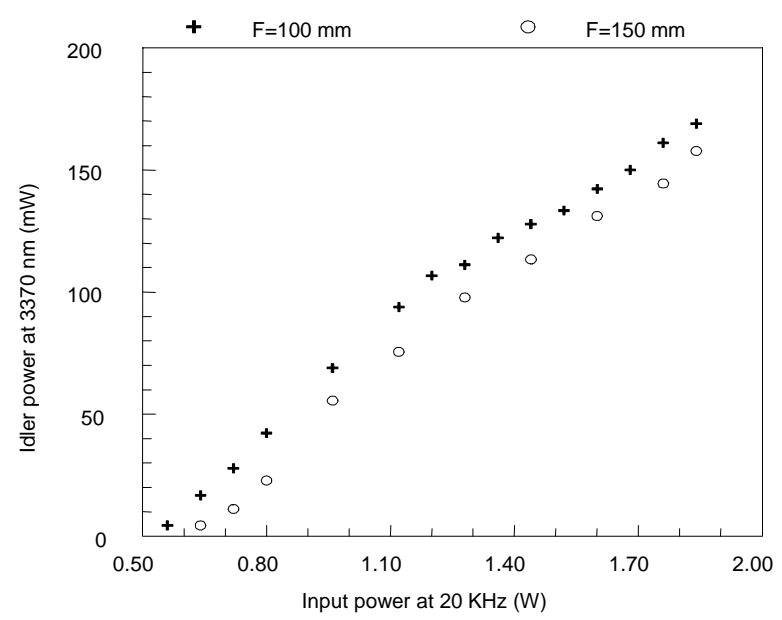

Figure 6 Laser output at $3370 \mathrm{~nm}(29.4 \mu \mathrm{m}$ period and $190 \mathrm{C})$.

The input power in Figure 6 is the actual power entering the input coupler of the OPO, and about $80 \%$ of the output of the laser. The $3374 \mathrm{~nm}$ idler power is corrected for the losses of a long-wave-pass filter (10\%), separating the idler from the pump and the signal. In addition to the idler power at $3374 \mathrm{~nm}$, also the signal power at $1555 \mathrm{~nm}$ was measured. The results with the $F=100 \mathrm{~mm}$ lens can be seen in Figure 7. At present, it is unclear why the two curves start to deviate above an input power of $1.2 \mathrm{~W}$.

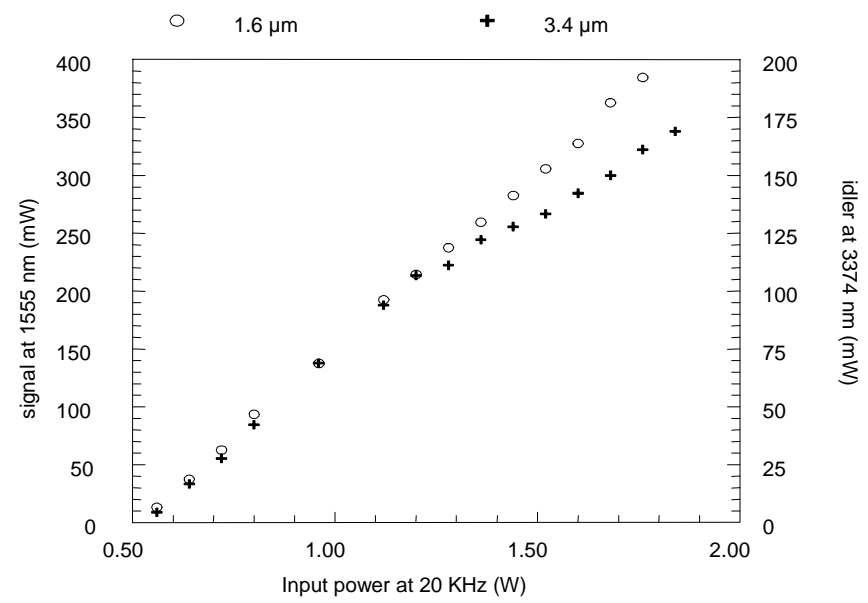

Figure 7 Laser output for $F=100 \mathrm{~mm}$.

The threshold pump laser power is approximately $0.5 \mathrm{~W}$ (at $20 \mathrm{kHz}$ ) or $25 \mu \mathrm{J}$. If we use the measured focus diameter of $118 \mu \mathrm{m}$, we arrive at a threshold energy density of $0.23 \mathrm{~J} / \mathrm{cm}^{2}$, and a threshold power density of $27.9 \mathrm{MW} / \mathrm{cm}^{2}$. The power efficiency is $8.8 \%$ (idler only) and the slope efficiency is $15 \%$ (idler only). In the table below, we compare the results with the values found in the literature review.

Table 6 TNO laser ccompared with literature values.

\begin{tabular}{lll}
\hline Property & TNO laser & Literature \\
\hline Threshold energy density & $0.23 \mathrm{~J} / \mathrm{cm}^{2}$ & $0.15-0.21 \mathrm{~J} / \mathrm{cm}^{2}$ \\
Threshold power density & $27.9 \mathrm{MW} / \mathrm{cm}^{2}$ & $4.5-18 \mathrm{MW} / \mathrm{cm}^{2}$ \\
Power efficiency (idler) & $8.8 \%$ & $7.5-13 \%$ \\
Slope power efficiency (idler) & $15 \%$ & $11-22 \%$ \\
\hline
\end{tabular}

The efficiencies compare very well with the literature ${ }^{4-11}$. The threshold energy and power density, however, are higher than any literature value found during our literature review, but this can be attributed to low reflection for the signal 
beam at the output coupler. Also these values critically depend on the focus diameter, which was hard to measure with our current set-up. The power efficiency and slope power efficiency measurements are more straightforward and reliable, and assure us that we have built a state-of-the-art mid-IR laser.

To further increase the average power of the laser and therefore simultaneously increase the jam to signal ratio we must increase the pulse frequency. However, this result in lower pump pulse energy and increased pump pulse length, resulting in lower peak powers (see Table 4). In Table 7 the results of the output power measurements at higher reprates is shown.

Table 7 Output at higher rep-rates $(\mathrm{F}=100 \mathrm{~mm})$.

\begin{tabular}{llllll}
\hline $\begin{array}{l}\text { Pulse } \\
\text { frequency } \\
(\mathrm{KHz})\end{array}$ & Input power & \multicolumn{2}{l}{$\begin{array}{l}\text { Signal energy/power } \\
(1555 \mathrm{~nm})\end{array}$} & \multicolumn{2}{l}{$\begin{array}{l}\text { Idler energy/power } \\
(3374 \mathrm{~nm})\end{array}$} \\
\hline 20 & $(\mathrm{~W})$ & $(\mu \mathrm{J})$ & $(\mathrm{mW})$ & $(\mu \mathrm{J})$ & $(\mathrm{mW})$ \\
22 & 1.88 & 19 & 380 & 7.90 & 158 \\
24 & 2.00 & 17.4 & 382 & 7.36 & 162 \\
26 & 2.12 & 16.7 & 400 & 6.96 & 167 \\
28 & 2.24 & 15.7 & 408 & 6.46 & 168 \\
30 & 2.36 & 14.6 & 410 & 6.07 & 170 \\
\hline
\end{tabular}

Although above $20 \mathrm{kHz}$ the pulse energy decreases, there is an increase in average power for the idler up to about $28 \mathrm{kHz}$.

\subsection{4 micron generation}

For DIRCM applications it is very important to generate jam power in the 3-5 $\mu \mathrm{m}$ band. It is of interest how far we can push the system to generate as much power as possible and to emit jam wavelength for the largest wavelength range as possible. Of special interest is to know how closely we can approach the 5 micron wavelength. With the current grating periods and for a crystal temperature of $190^{\circ} \mathrm{C}$ we can reach at most $3866 \mathrm{~nm}$. In principle, we could use lower crystal temperatures, thus increasing the maximum wavelength, but this will decrease the lifetime of the crystal, according to literature reports ${ }^{12}$. In the Table 8 and Figure 8 we have plotted the generated jam power as function of the wavelength.

Table 8 Idler output power with $1.88 \mathrm{~W}$ pump input power $(20 \mathrm{kHz})$ for grating periods of 28.2, 28.6, 29.0 and $29.4 \mu \mathrm{m}$ for crystal temperature of $190^{\circ} \mathrm{C}$.

\begin{tabular}{cccc}
\hline Idler wavelength $(\mathrm{nm})$ & Grating period $(\mu \mathrm{m})$ & Measured power idler $(\mathrm{mW})$ & Photon number \\
\hline 3866 & 28.2 & 131 & $2.55 .10^{18}$ \\
3697 & 28.6 & 150 & $2.79 .10^{18}$ \\
3541 & 29.0 & 153 & $2.73 .10^{18}$ \\
3374 & 29.4 & 158 & $2.68 .10^{18}$ \\
\hline
\end{tabular}

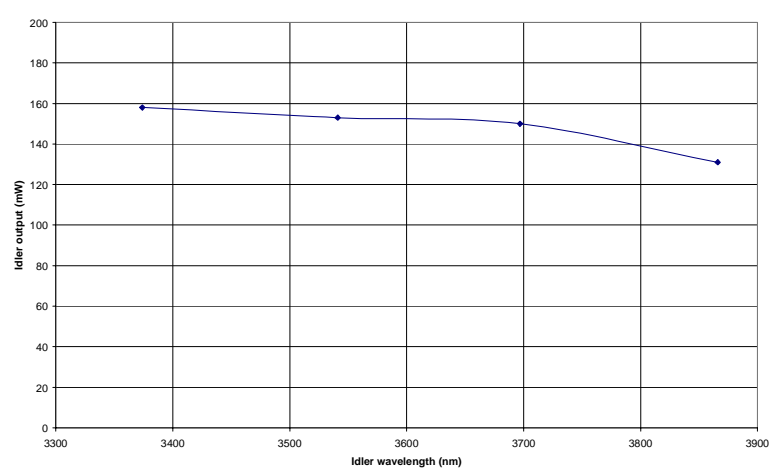

Figure 8 Idler output power with 1.88 W pump input power (20 kHz) for grating periods of 28.2, 28.6, 29.0 and 29.4 $\mu \mathrm{m}$ for crystal temperature of $190^{\circ} \mathrm{C}$. 
The power decreases for longer idler wavelengths. Although the PPLN crystal absorption increases for longer wavelength, this is not an absorption effect since the photon number increases at first. We notice a sudden drop for the $28.2 \mu \mathrm{m}$ period, which seems larger than expected for a gradual increase in PPLN absorption as function of wavelength. A possible explanation is that there is a variation in the transmission of the long-wave pass filter, used to separate the idler from the pump and signal, which should be further looked into.

\subsection{2 micron generation}

With the longest period of the PPLN crystal $(31.0 \mu \mathrm{m})$ it is possible to have a signal $(2050 \mathrm{~nm})$ and idler $(2212 \mathrm{~nm})$ with almost identical wavelength at approx. $175^{\circ} \mathrm{C}$ (see Figure 9).

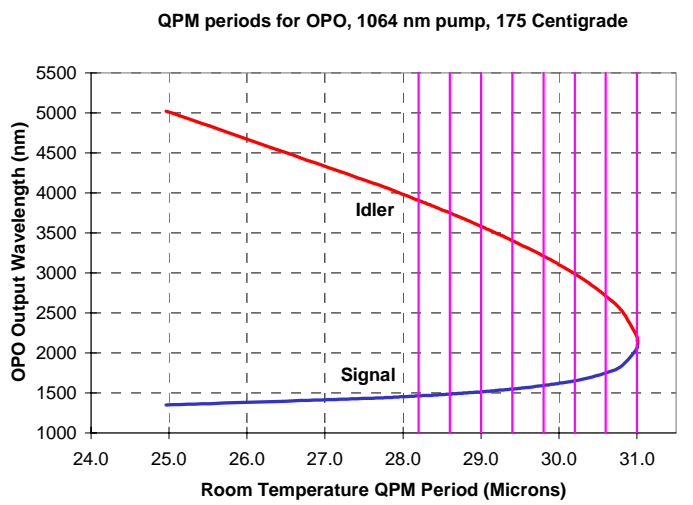

Figure 9 Wavelength with PPLN crystal at $175^{\circ} \mathrm{C}$.

The total output (signal+idler) from the OPO was measured at different temperatures with $1.88 \mathrm{~W}$ input power at $20 \mathrm{kHz}$ rep-rate and $F=150 \mathrm{~mm}$, as depicted in Figure 10.

As expected, above about $175^{\circ} \mathrm{C}$ the output rapidly decreases because the grating periods become too long for phase matching.

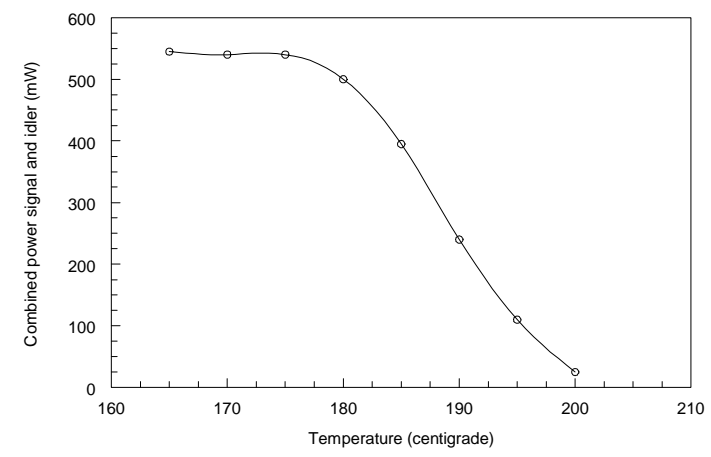

Figure 10 Output signal and idler with $31.0 \mu$ m grating period.

Using the knife-edge method ${ }^{13}$, the beam quality parameter $\left(M^{2}\right)$ of the idler output of the laser was measured as well (for which a grating period of $29.4 \mu \mathrm{m}$ was used). The idler beam $(3.37 \mu \mathrm{m})$ was collimated with a $\mathrm{CaF}_{2}$ lens $(F=95 \mathrm{~mm})$ and focused with a germanium lens $(F=50 \mathrm{~mm})$. The transmission of the germanium lens, which was coated for $10.6 \mu \mathrm{m}$ is only about $50 \%$ for the idler wavelength. This reduces the power on the detector. The most sensitive range of the thermopile detector is $100 \mathrm{~mW}$ full scale. The beam focus measurements were even less reliable than the previous beam focus measurements of the pump laser. This time we only had approx. $50 \mathrm{~mW}$ power available for the 
knife-edge measurements and at the same time we had to measure with a thermopile detector with an inherent slow response.

For a focal length of $50 \mathrm{~mm}$ the diameter of the focus for a laser with $M^{2}=1$ would be $45 \mu \mathrm{m}$. The smallest focus diameter that was measured was $124 \mu \mathrm{m}$, resulting in a $M^{2}$ of 2.5 . With an accuracy of $40 \mu \mathrm{m}$ of the focus diameter the $M^{2}$ would be $2.5 \pm 0.9$.

Because of the small depth of focus (DOF) the knife must be placed almost exactly at the focus position or a too large diameter will be measured. If the knife-edge were placed $0.5 \mathrm{~mm}$ from the focus position the measured focus diameter would be about 1.5 times larger. This would mean that the calculated $M^{2}$ would be about 1.5 times smaller and would be between 1.1 and 2.3. The set-up used to measure the beam quality needs to be improved for more accurate determination of the beam quality parameter $M^{2}$.

\subsection{Jamming to signal calculation}

We can calculate the jamming to signal ratio, that we can achieve with our current laser. We assume we have $160 \mathrm{~mW}$ of jam power in the whole wavelength range from 2 to 4 microns, although at approx. $2 \mu \mathrm{m}$ we will have a boost in output power as the signal and idler wavelengths are then identical. This is less than the operational required jam power of 1 Watt with $1 \mathrm{mrad}$ beam divergence or 4 Watt with 2 mrad beam divergence. To compensate for the reduced laser power we will more tightly focus our beam. Since the laser will be used in laboratory and field experiments, to study infrared countermeasure techniques, we can more easily pin point the beam to the target in comparison to an operational system. We will use an optical output beam diameter of either $15 \mathrm{~mm}$ or $25 \mathrm{~mm}$, and we assume that there will be an optical loss of $30 \%$ in lenses and windows. Based on these assumptions the $J / S$ ratio is calculated and plotted in Figure 11 for an optical aperture of 15 and $25 \mathrm{~mm}$, respectively, as function of beam divergence. The lowest beam divergence corresponds to a jammer wavelength of 2 micron, the largest beam divergence corresponds to a jammer wavelength of 4 micron.

We can see that for aircraft signatures in the range from 10 up to $1000 \mathrm{~W} / \mathrm{sr}$ and for an optical aperture of $15 \mathrm{~mm}$ or 25 $\mathrm{mm}$ we can maintain a $J / S$ ratio of more than $23 \mathrm{~dB}$ for all conditions. The lowest $J / S$ ratio is achieved for the highest aircraft thermal signature of $1000 \mathrm{~W} / \mathrm{sr}$ and for a wavelength of 4 micron. In this case, we reached a $J / S$ ratio of $23 \mathrm{~dB}$ and $27 \mathrm{~dB}$ for an optical aperture of 15 and $25 \mathrm{~mm}$ respectively. This is below our target figure of $30 \mathrm{~dB}$.

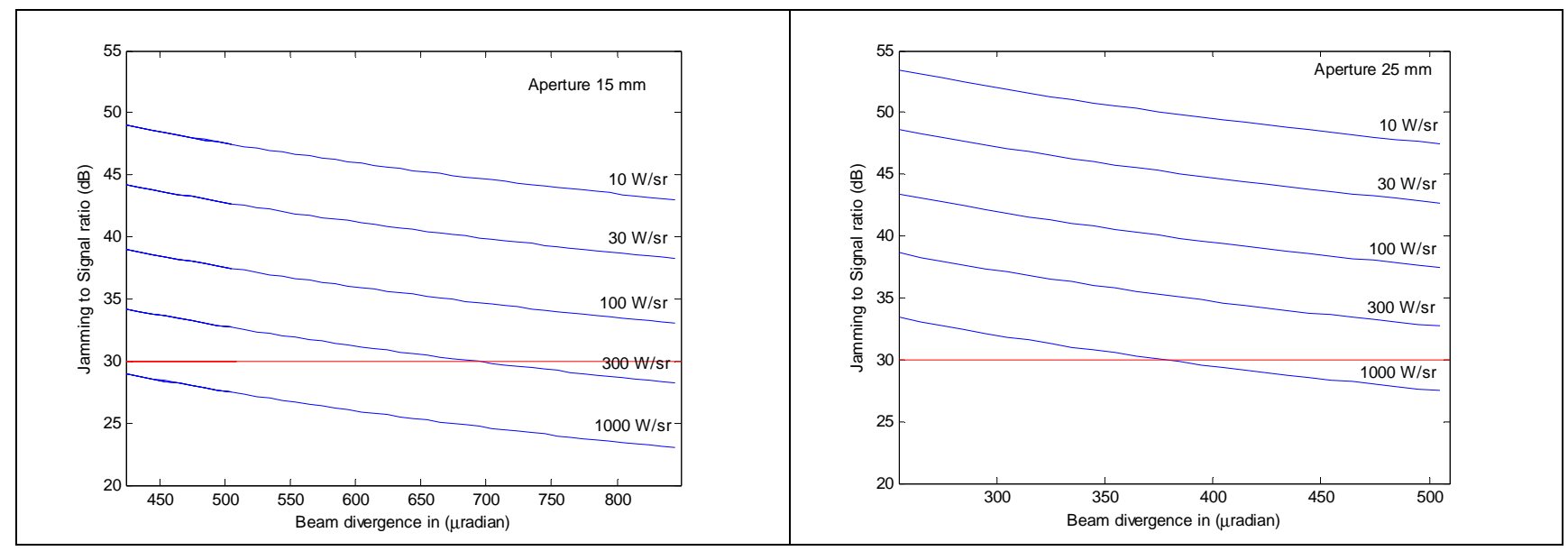

Figure 11 (Left) Laser power 0.16 Watt, optical transmission 0.7, $M^{2}=2.5$. Beam divergences used that correspond with an aperture of $15 \mathrm{~mm}$ for a wavelength varying between 2 and $4 \mu \mathrm{m}$; Laser power 0.16 Watt, optical transmission $0.7, M^{2}=2.5$. Beam divergences used that correspond with an aperture of $25 \mathrm{~mm}$ for a wavelength varying between 2 and $4 \mu \mathrm{m}$. 


\subsection{Laser jam pattern characterisation}

To be able to jam IR missiles a modulation pattern must be generated. We used an acousto-optic modulator AOM.

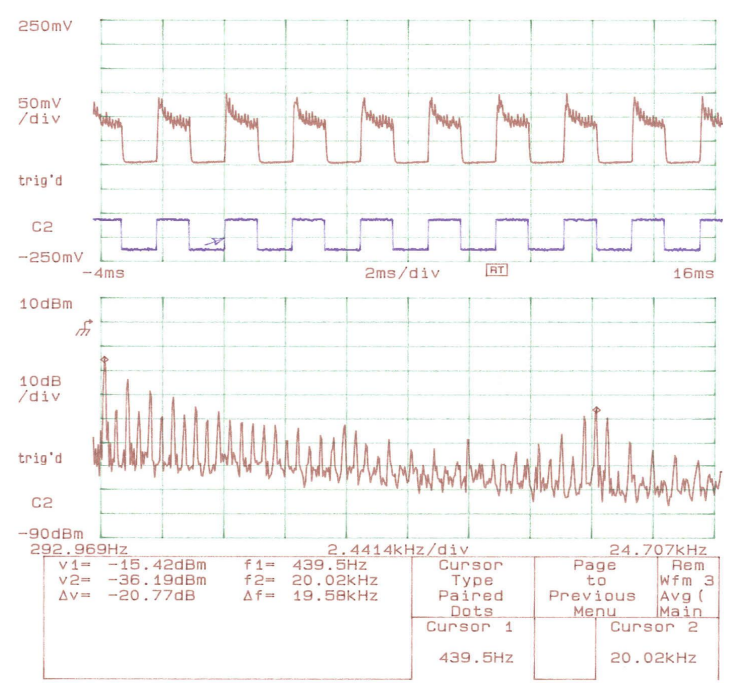

Figure 12 Modulated laser output (top curve), modulation pattern (middle curve) and Fourier spectrum of modulated laser output (bottom curve).

When an RF frequency is put on the AOM, it generates a bulk acoustic wave in the modulator crystal. The laser beam will be diffracted at the induced grating and will be deflected to another direction. The AOM can diffract about $90 \%$ of the laser energy. We have placed the AOM behind the beam expander. If no RF pulse is fed into the AOM the pump laser pulse will travel undeflected by the AOM into the OPO cavity and signal and idler wavelengths will be generated. If an RF pulse is fed into the modulator, $90 \%$ of the pump laser pulse will be deflected and it will not reach the OPO cavity, but it will be dumped into a beam dump. Only $10 \%$ of the pump laser beam reaches the OPO cavity, but since this power is below the threshold for oscillation no signal and idler wavelengths will be generated. Therefore, a 100\% modulation of the idler wavelength (the jam laser wavelength) can be achieved. The RF-power to the AOM can be modulated by a 5 V TTL input signal up to several MHz.

To measure the modulated laser output (idler OPO output) an uncooled $1 \mathrm{GHz} \mathrm{HgCdTe}$ detector was used. This detector was placed after the long-wave pass filter used to separate the idler wavelength from the pump and signal wavelengths. The AOM was modulated with a $440 \mathrm{~Hz}$ square wave (see the middle curve of Figure 12). The laser was working at maximum power at a rep-rate of $20 \mathrm{kHz}$. The output from the $\mathrm{HgCdTe}$ detector was filtered $(0.1 \mathrm{~Hz}-10 \mathrm{kHz})$ and amplified and is shown in Figure 12 (top curve). We recognise the square modulation pattern. When the laser is on (top of the curves of the top curve), we can almost observe the individual pump laser pulses. It also appears as if the power drops within a half period of the modulation square wave, but this is an artefact since the detector was AC coupled. In addition to the $440 \mathrm{~Hz}$ signal (and many higher harmonics), the $20 \mathrm{kHz}$ rep-rate of the laser is also visible in the FFT spectrum (bottom curve in Figure 12). Despite the fact that the $\mathrm{HgCdTe}$ detector signal was filtered with a bandpass filter between $0.1 \mathrm{~Hz}$ and $10 \mathrm{kHz}$, some $20 \mathrm{kHz}$ still passed.

We have thus successfully shown that IR jamming pulses with various patterns can effectively be created using the AOM modulator.

\section{CONCLUSIONS}

In this project, a tuneable mid-infrared laser source was built. The system was composed of commercial of the shelf components. The power efficiency $(8.8 \%)$ and slope power efficiency $(15 \%)$ measurements indicate that our laser source can compete with state of the art mid-IR laser sources (see Table 6). 
At present, wavelengths in the range of 1.5 to $3.9 \mu \mathrm{m}$ can be generated. The maximum wavelength that can be generated is limited by the grating period in our PPLN crystal and by the fact that the crystal has to be kept at elevated temperatures $\left(>180^{\circ} \mathrm{C}\right)$ to avoid permanent photorefractive damage. Another crystal with different grating periods would allow us to increase the maximum jammer wavelength. However, above $4 \mu \mathrm{m}$ the non-linear PPLN crystal starts to absorb the generated radiation, thus reducing the idler output power levels.

Power levels vary across the wavelength range from $400 \mathrm{~mW}$ at $1.5 \mu \mathrm{m}$ to $130 \mathrm{~mW}$ at $3.9 \mu \mathrm{m}$. The output power can be further increased with a more powerful pump laser. The current power levels allow us to successfully perform groundbased seeker jamming experiments. We have calculated that for an optical aperture of $15 \mathrm{~mm}$ and $25 \mathrm{~mm}$ we can reach a $J / S$ ratio of more than $23 \mathrm{~dB}$ in all situations for aircraft signatures in the range of $10-1000 \mathrm{~W} / \mathrm{sr}$.

We have also shown that with incorporation of an acousto-optic modulator we can generate all kinds of complicated jam sequences.

\section{ACKNOWLEDGEMENT}

The authors would like to thank the financial support received from Royal Netherlands Air Force under contract DMKLu/20/121/03/7019/01.

\section{REFERENCES}

1. L. Sjöqvist, O. Steinval, D. Titterton, V. Michau, R. Schleijpen, CEPA JP8.11 "Laser Beam Steering”, WP2 report, 2003.

2. H.M.A. Schleijpen, TNO report FEL-03-A190, "DIRCM System Analysis", 2003.

3. G. Arisholm, K. Stenersen, G. Rustad, E. Lippert, M. Haakestad, "Mid-infrared optical parametric oscillators based on periodically-poled lithium niobate”, FFI report 02830, 2002.

4. V. Sirutkaitis, O. Balachninaite, A. Atamalian, R. Grigonis, R.C. Eckardt, "Periodically poled lithium niobate optical parametric oscillator pumped by a diode-pumped, Q-switched Nd:YAG laser", SPIE Vol. 4751, 65 (2002).

5. C. Drag, I. Ribet, M. Jeandron, M. Lefebvre, E. Rosencher, "Temporal behavior of a high repetition rate infrared optical parametric oscillator based on periodically poled materials", Appl. Phys. B73, 195-200 (2001).

6. I.F. Elder, J.A.C. Terry, "Efficient conversion into the near- and mid-infrared using a PPLN OPO", J. Opt. A:Pure Appl. Opt. 2, L19-L23 (2000).

7. D.Y. Shen, S.C. Tam, Y.L. Lam. T. Kobayashi, "Singly resonant optical parametric oscillator based on periodically poled MgO:LiNbO3”, Electr. Lett. 36(17), 1488-1489 (2000).

8. L.E. Myers, W.R. Bosenberg, 'Periodically poled lithium niobate and quasi-phase-matched optical parametric oscillators', IEEE J. Quant. Electr. 33, 1663 (1997).

9. G.D. Miller, 'Periodically poled lithium niobate: modelling, fabrication, and nonlinear-optical performance', Dissertation Stanford University, July 1998.

10. D. Matthews, L.R. Marshall, 'Six-wavelength PPLN OPO’, OSA TOPS Vol. 10 Advanced Solid State Lasers, 244 (1997).

11. G. Sepp, R. Protz, 'Laser beam source for a directional infrared countermeasures (DIRCM) weapon system', Patent no.:US 6,587,486 B1, July 1, 2003.

12. M.M.J.W. van Herpen, PhD Thesis Katholieke Universiteit Nijmegen, "Continuous-wave optical parametric oscillator for trace gas detection in life sciences", (2004).

13. J.C. van den Heuvel, F.J.M. van Putten, R.J.L. Lerou, "The SRS Threshold for a nondiffraction-limited pump beam”, IEEE J. Quant. Electr. 28 (9), 1930-1936 (1992). 